\title{
Cognitive Emotion Regulation Strategies Profile in Students with High and low Interpersonal Sensitivity
}

\author{
Zahra Ahmadi ${ }^{1}$, Shole Amiri ${ }^{2, *}$, Mehrdad Kalantari ${ }^{3}$, Mohammad Reza Abedi ${ }^{4}$, Hossein Molavi ${ }^{5}$ \\ ${ }^{1}$ Ph.D. Student of Psychology, University of Isfahan, Isfahan, Iran \\ ${ }^{2}$ Professor, Department of Psychology, University of Isfahan, Isfahan, Iran \\ ${ }^{3}$ Professor, Department of Psychology, University of Isfahan, Isfahan, Iran \\ ${ }^{4}$ Professor, Department of Counseling, University of Isfahan, Isfahan, Iran \\ ${ }^{5}$ Professor, Department of Psychology, University of Isfahan, Isfahan, Iran
}

\section{ARTICLE INFO}

\section{Article History}

Received:: 8 December 2019

Revised: 7 November 2020

Accepted: 16 November 2020

Available online: February 2021

Article Type

Research Article

Keywords

Interpersonal sensitivity; Emotion regulation; Cognitive emotion regulation strategies; Students

\section{Corresponding Author*}

Shole Amiri received her Ph.D. in Psychology from University of Tehran. She is currently a Professor in Department of Psychology at University of Isfahan. Her research interests are in developmental Psychology.

Correspondence concerning this article should be addressed to dr. Amiri, Department of Psychology Faculty of Education and Psychology, University of Isfahan, Azadi square, University Ave., Isfahan Postal Code 81746-7344.

ORCID: 0000-0002-3914-1244

E-mail: s.amiri@edu.ui.ac.ir

doi: http://dx.doi.org/10.29252/bjcp.14.2.15

\section{ABSTRACT}

The purpose of this study was to compare the cognitive emotion regulation strategies profile in students with high and low interpersonal sensitivity.

The descriptive and survey method was used. The statistical population was all undergraduate students of the University of Isfahan in the academic year 2018-2019. According multistage cluster random sampling 314 students were selected as samples and they were asked to fill in the Symptom Checklist 90 Revised and Cognitive Emotion Regulation Questionnaire. The data were analyzed by the discriminant analysis and multiple correlation method.

Findings showed that among 9 investigated strategies in two groups of students with high and low interpersonal sensitivity, the catastrophizing, other-blame, rumination, acceptance were significant $(\mathrm{P}<0.001)$. Also, the differences in re-focusing on planning, positive re-evaluation, self-blame, and positive refocusing between two groups were significant $(\mathrm{P}<0.05)$. Considering the small amount of Wilks Lambda and the high amount of the Chi-square and significance level, the audit function has obtained a good diagnostic power for explaining the dependent variable.

Based on all cognitive emotion regulation strategies (except putting into perspective), the profile of students with high and low interpersonal sensitivity can be separated. 


\title{
نيمرخ راهبر دهاى تنظيم شناختى هيجان در دانشجويان با حساسيت بينفردى بالا و پايين
}

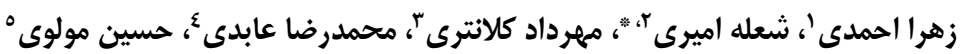

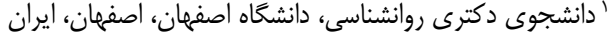

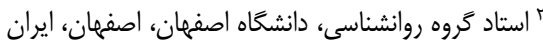

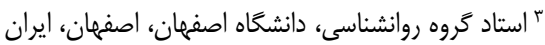

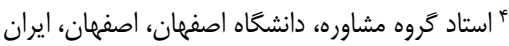

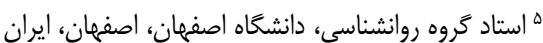

\section{يكيده}

اطلاعات مقاله

هدف از اين يزوهش مقايسهى نيمرخ راهبردهاى تنظيم شناختى هيجان در دانشجويان با حساسيت بينفردى بالا و پايين بود.

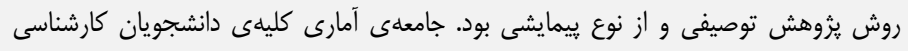

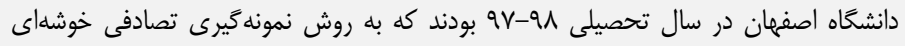

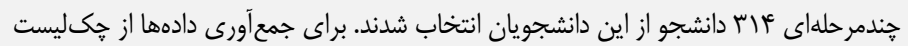
تجديدنظرشدهى نشانههاى روانى و يرسشنامهى تنظيم شناختى هيجان و براى تحليل دادئ دادها

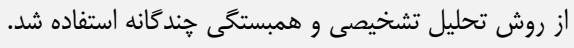

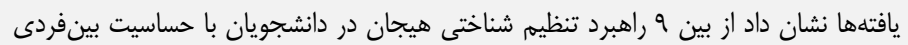

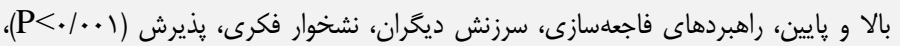

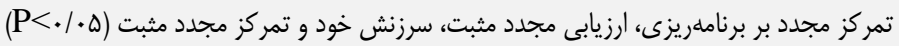

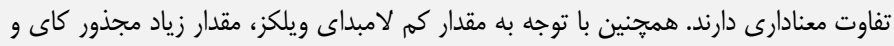

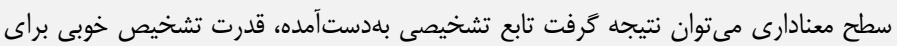
تمايز متغير وابسته دارد.

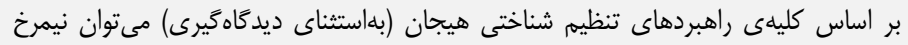

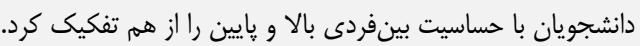

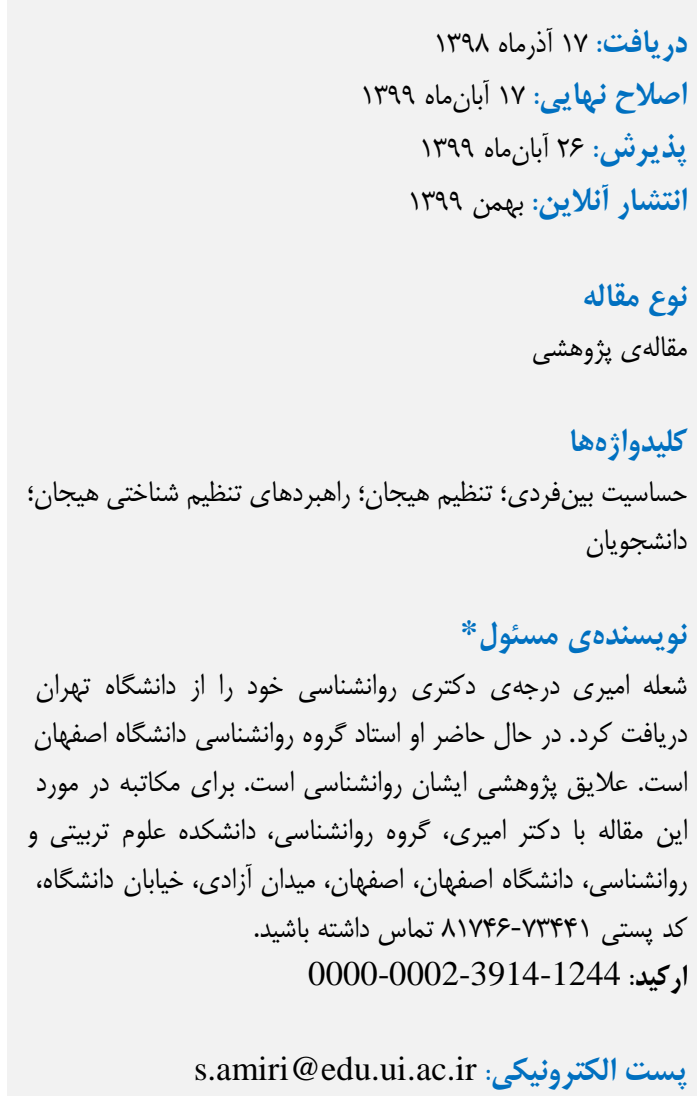

يست الكترونيكى: s.amiri@edu.ui.ac.ir

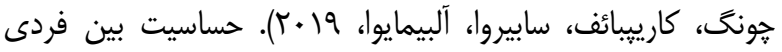

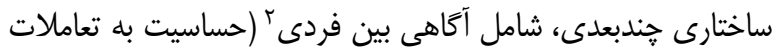

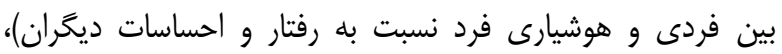

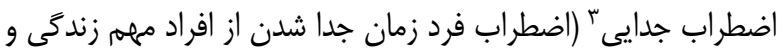

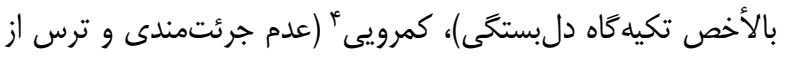

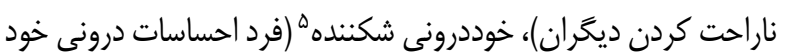
را به خاطر ترس از طرد شدن و مورد انتقاد قرار كَرفتن بيان نمى كنداند) و نياز به تأييد (تمايل فرد براى خشنود كردن و رضايت ديكران) است
حساسيت بين فردى' به معناى آكاهى و حساسيت بيشازحد، نسبت به رفتار و احساسات ديگران است كه با احساس نار ناراحتى،

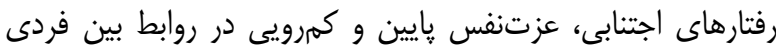

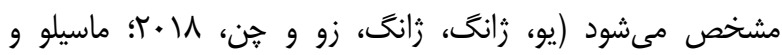

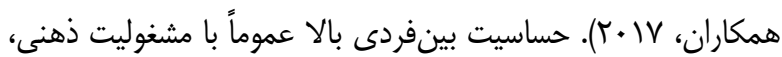

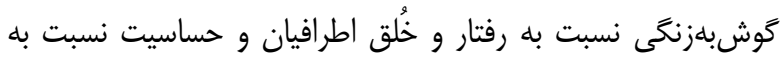
دريافت انتقاد و طرد در تعاملات اجتماعى همراه است (اسلانبكوا،
${ }^{4}$ Timidity
${ }^{5}$ Fragile inner self
${ }^{6}$ Need to be confirmed

\footnotetext{
${ }^{1}$ Interpersonal sensitivity

${ }^{2}$ Interpersonal awareness

${ }^{3}$ Separation anxiety
} 
سرزنش خود" براى بررسى و يردازش رويدادهاى هيجانى، انجام

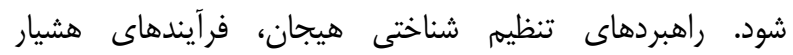

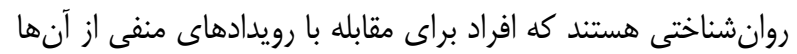

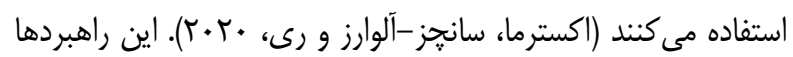

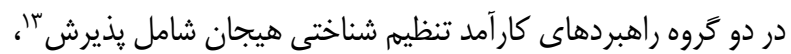
تمركز مجدد مثبت"

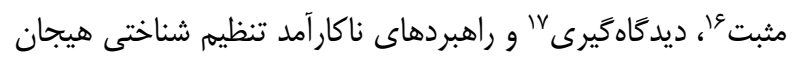

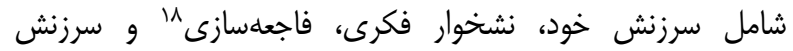

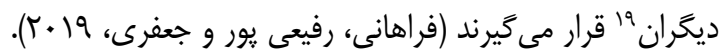

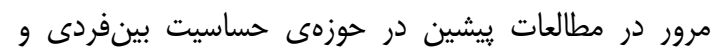

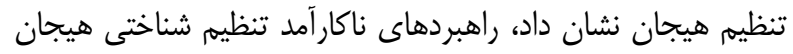

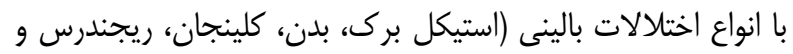

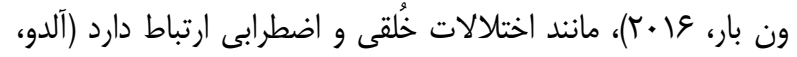

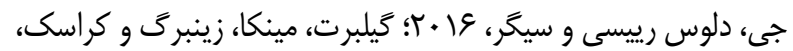

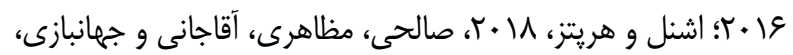

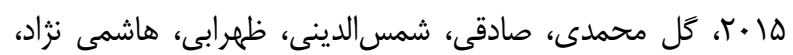

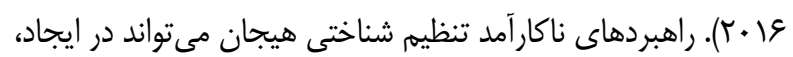

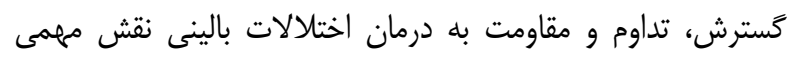

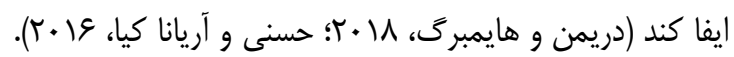

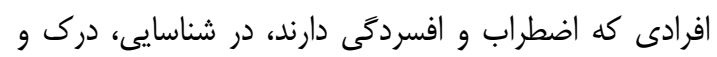

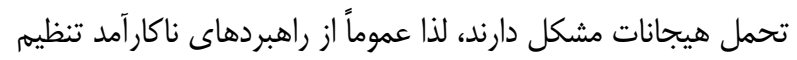

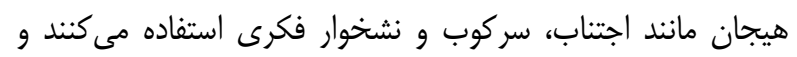

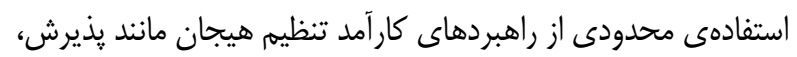

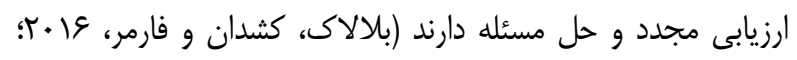

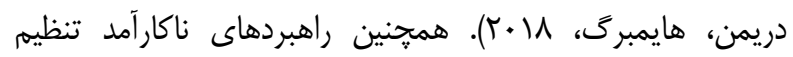

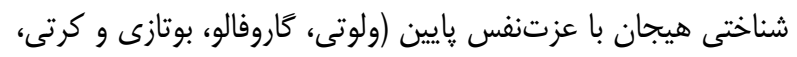

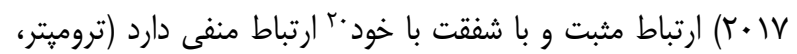

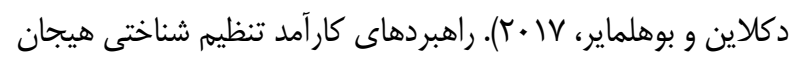
با افزايش عاطفهى مثبت و كاهش عاطفهى منفى (اودو و برينكر،

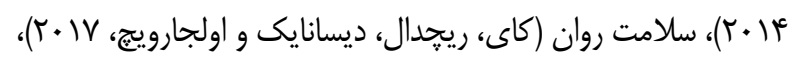

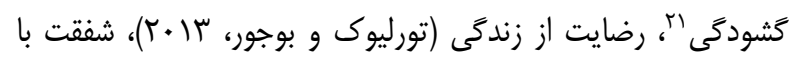

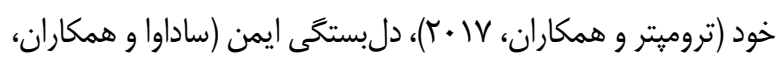

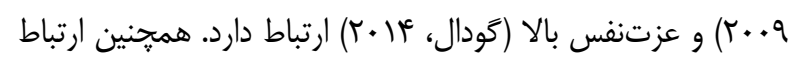

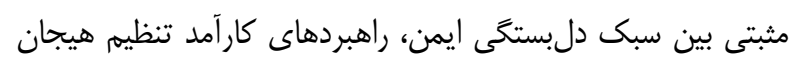

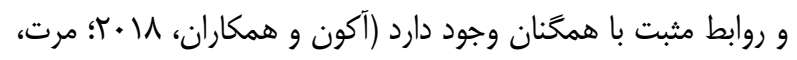

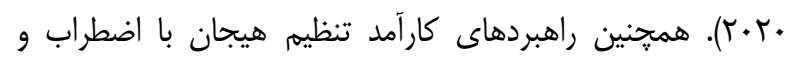

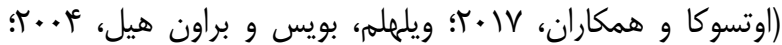

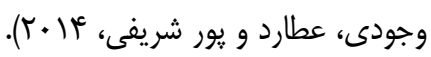

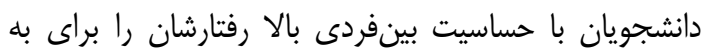
حداقل رساندن خطر سرزنش يا طرد، توسط انتظارات ديكران تعديل

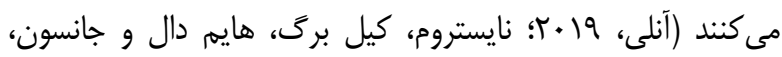

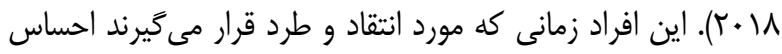

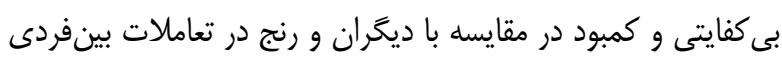

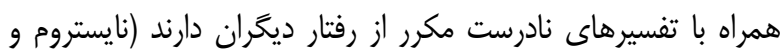

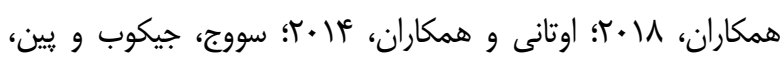

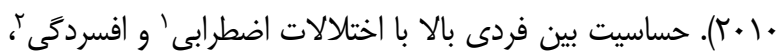

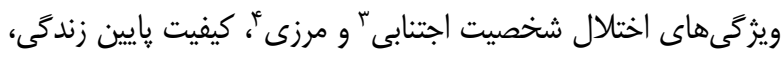

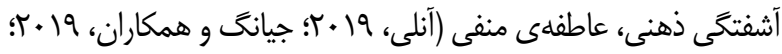

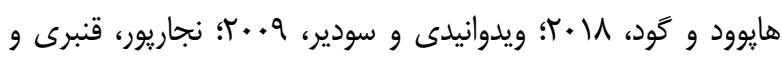

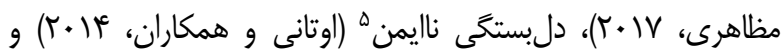

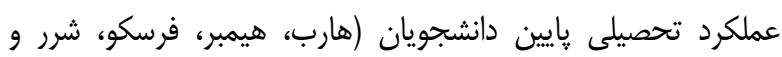

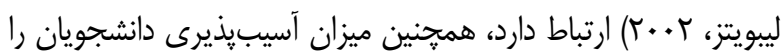

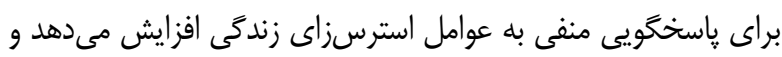

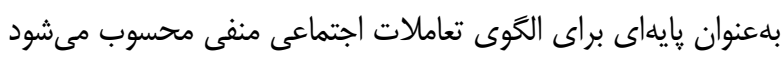

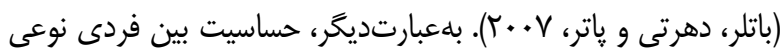

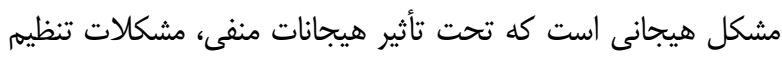

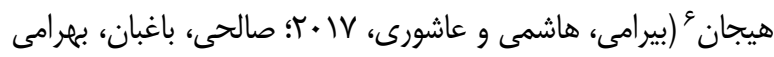

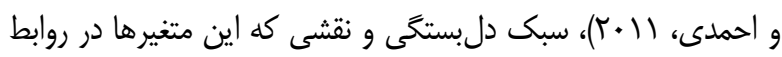

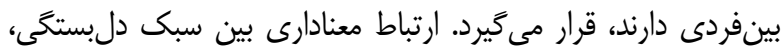

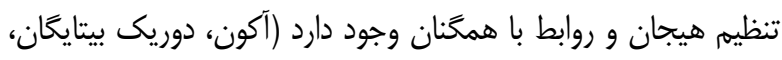

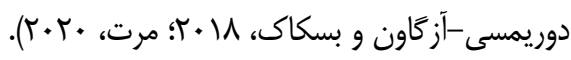

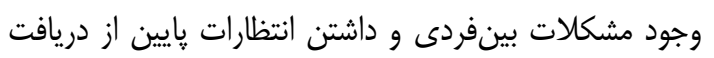

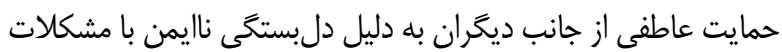

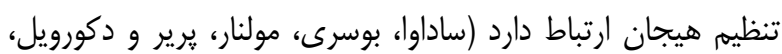

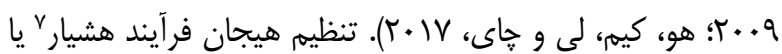

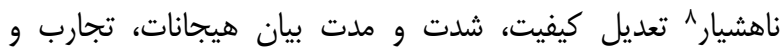

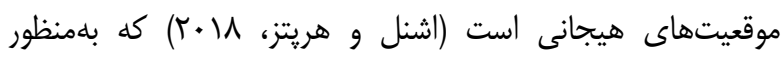

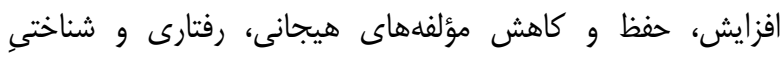

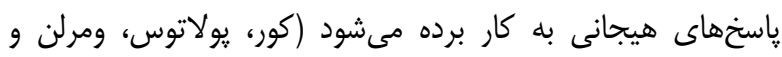

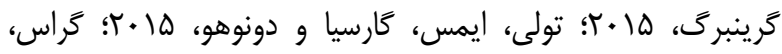

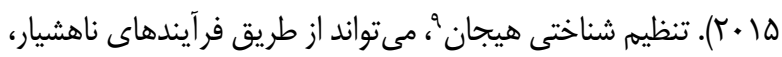

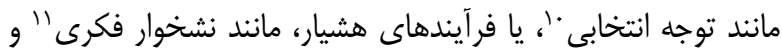

\footnotetext{
${ }^{12}$ Self-blame

${ }^{13}$ Acceptance

${ }^{14}$ Positive refocusing

${ }^{15}$ Planning refocusing

${ }^{16}$ Positive reappraisal

${ }^{17}$ Putting into perspective

${ }^{18}$ Catastrophizing

${ }^{19}$ Blaming others

${ }^{20}$ Self-compassion

21 Openness
}

\footnotetext{
1 Anxiety

${ }^{2}$ Depression

${ }^{3}$ Avoidant personality disorder

${ }^{4}$ Borderline personality disorder

${ }^{5}$ Attachment insecurity

${ }^{6}$ Emotion regulation

${ }^{7}$ Conscious

${ }^{8}$ Unconscious

${ }^{9}$ Cognitive emotion regulation

${ }^{10}$ Selective attention

${ }^{11}$ Rumination
} 
يُوهش را داشتند يرسشنامهها ارائه و ضمن تشكر از آنها خواسته

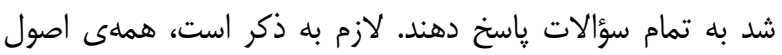

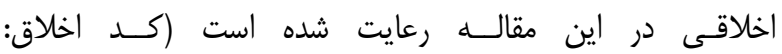

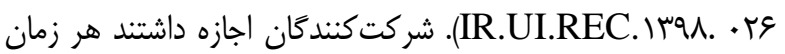

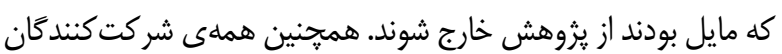

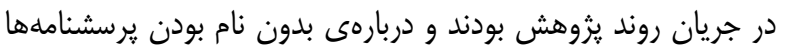

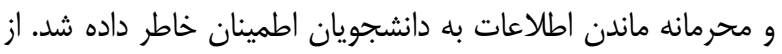

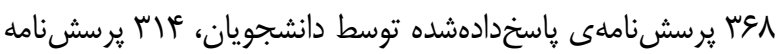

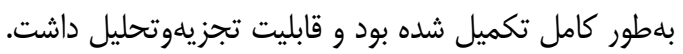

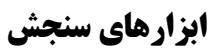

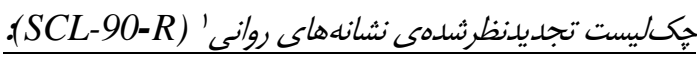

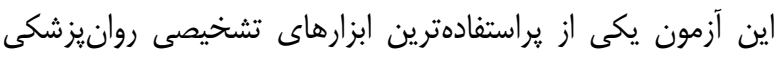

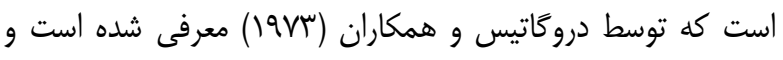

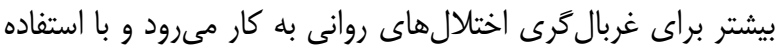

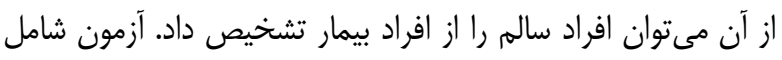

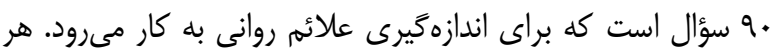

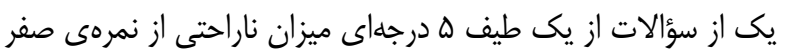

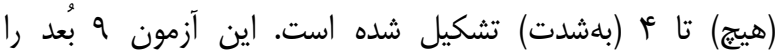

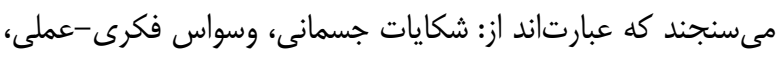

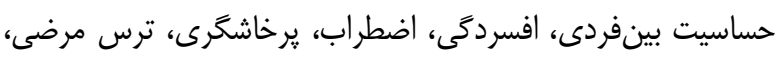

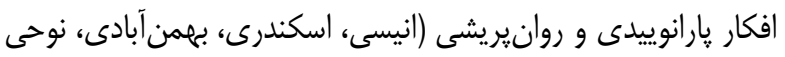

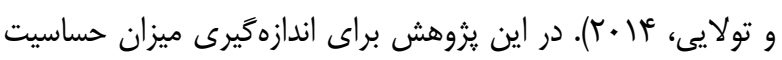

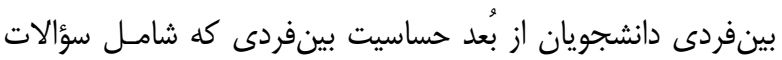
(1) سازندًان اين آزمون اعتبار درونى آن را با استفاده از ضريب استادي

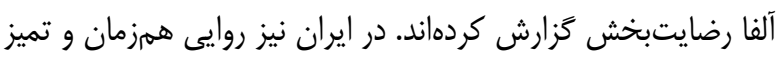

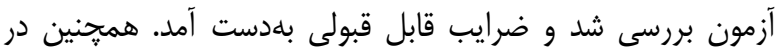

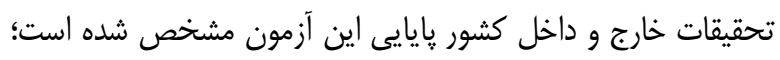

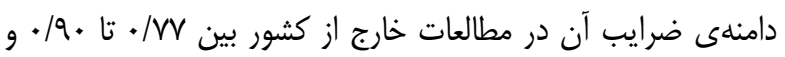

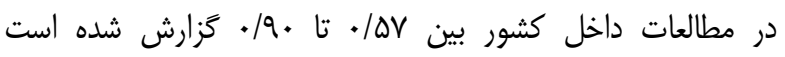

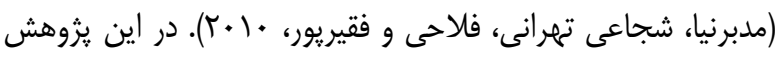

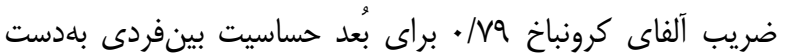

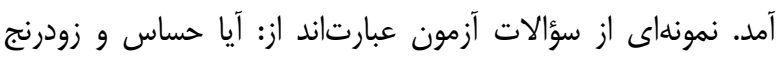

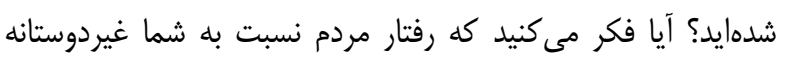
است يا آنكه شما را دوست ندارند؟

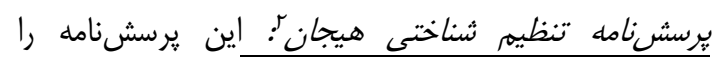

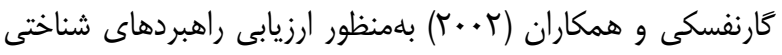

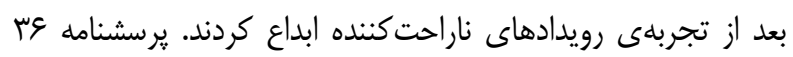

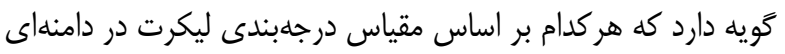

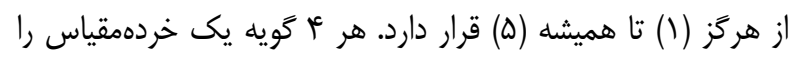

افسردگى ارتباط منفى دارند (ساس ورد، آلتن برگر، ماس، كرگ و و

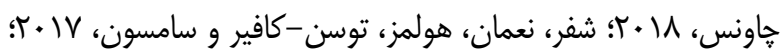

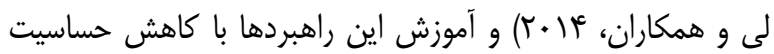

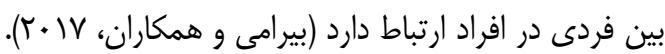

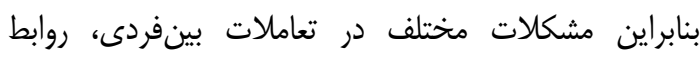
اجتماعى و مهارتهاى حل مسئله با راهبردهاى ناكارآمد تنظيم

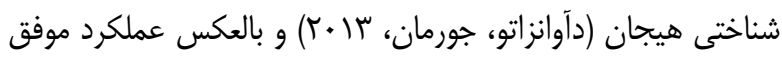

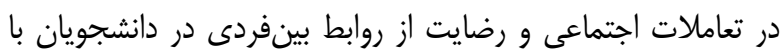

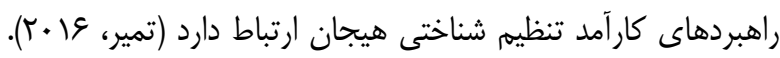

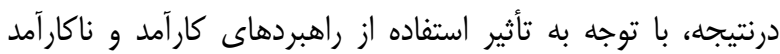

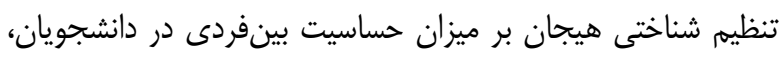

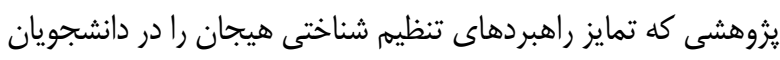

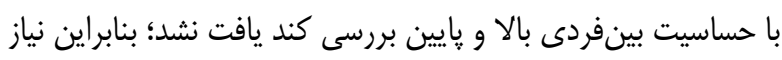

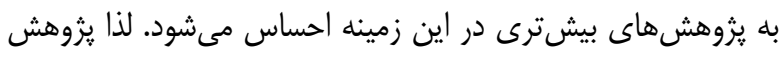

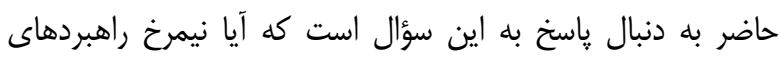

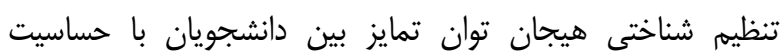

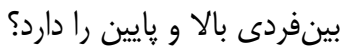

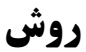

\section{طرح يُوهشى و شركتكنندكان}

طرح يزوهش توصيفى و از نوع بيمايشى بود. جامعلى آمارى،

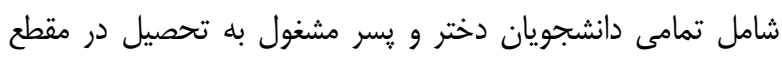
كارشناسى دانشكاه اصفهان، در سال تحصيلى

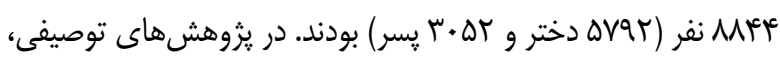

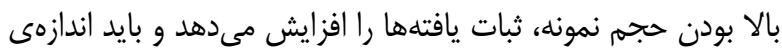

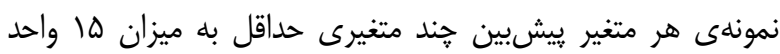

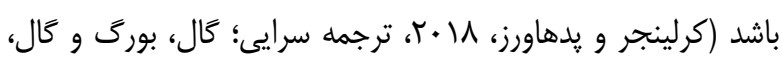

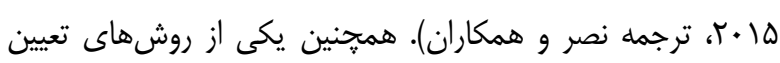
حجم نمونه در يزوهشهاى توصيفى استفاده از فرمول ار ائهشده توسط

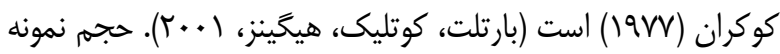

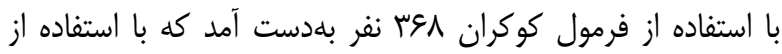

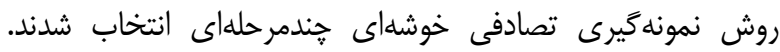

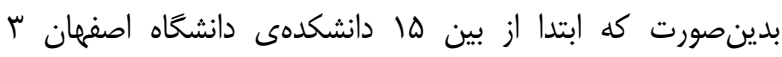

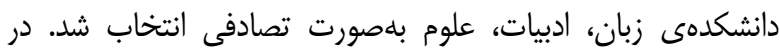

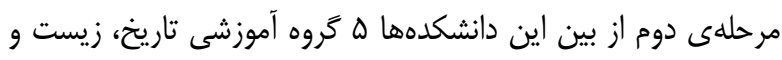

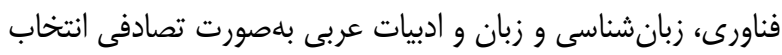

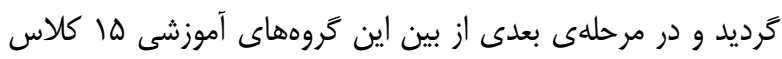
(از هر كروه آموزشى ب كلاس) بلهصورت تصادفى انتخاب شد.

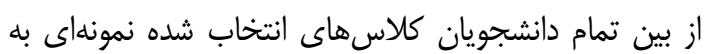

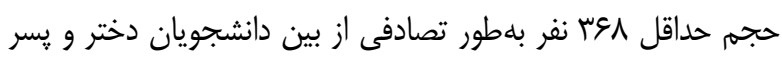
انتخاب شدند. به دانشجويانى كه داوطلبانه ابراز تمايل به شركت در در دران 
استفاده شد. به اين صورت كه از عاب دانشجويى كه به يرسشنامههاى

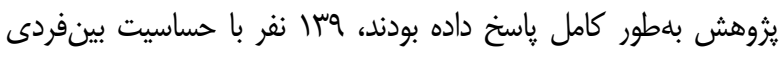

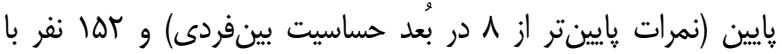

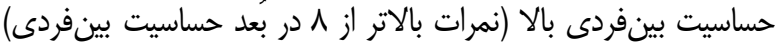

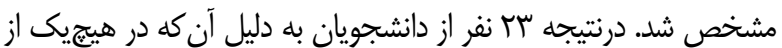

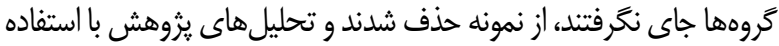
از از إن نفر دانشجو انجام شد.

بلهنظور تجزيهوتحليل دادها از نرمافزار بستهى آمارى در

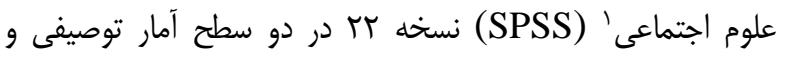
استباطى استفاده شد. جهت بررسى شاخص هاى توصيفى از ميانخين

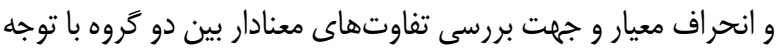

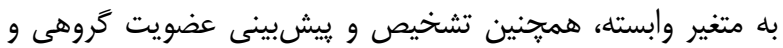

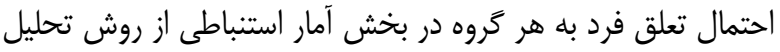

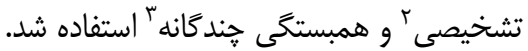

\section{نتايج}

ابتدا ييشفرضهاى تحليل تشخيصى مورد بررسى قرار كرفت. نتايج بررسى نرمال بودن با استفاده از نمودار هيستوكرام نشان داد داد كها

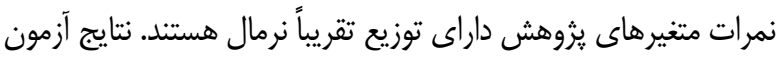

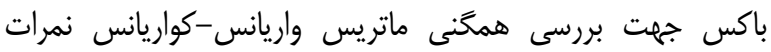

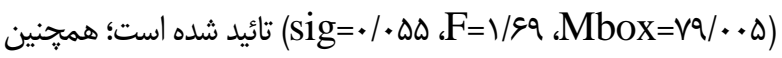
در متغيرهاى مستقل يزوهش (راهبردهاى شناختى تنظيم هيجان)

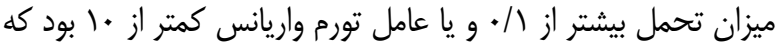
نشاندهندهى عدم همخطى بين متغيرهاى مستقل يثوهش هستند. در جدول 1 يافتهاى توصيفى، نتايج تحليل تشخيصى و و دري

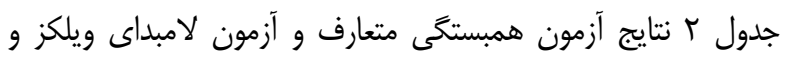
مجذور كاى براى بررسى تمايز راهبردهاى تنظيم شناختى هيجان در دو كروه دانشجويان با حساسيت بينفردى بالا و وإياين ارائه شده است.
تشكيل مىدهد و درمجموع 9 خردممقياس קذيرش، تمركز مجدد مثبت، تمركز مجدد بر برناملريزى، ارزيابى مجدد مثبت، ديدكاه

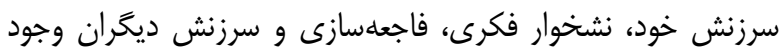

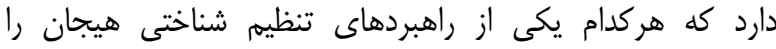

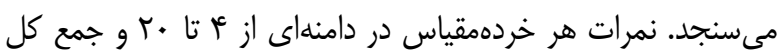

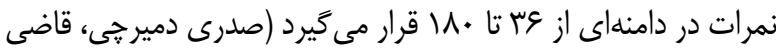

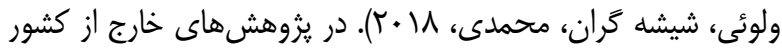

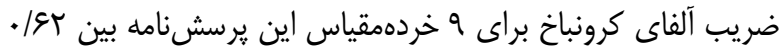

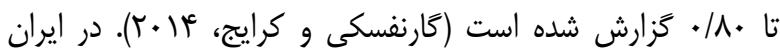

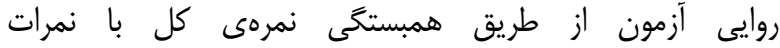

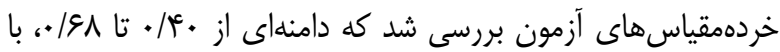
ميانگين \&ه/ • را دربرمى گرفت كه همخى معنادار بودند.

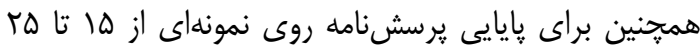

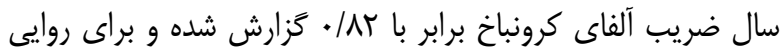

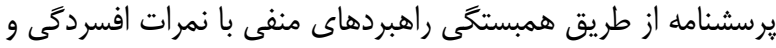

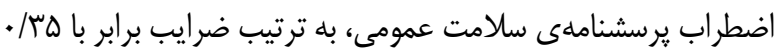

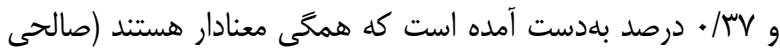

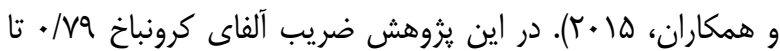

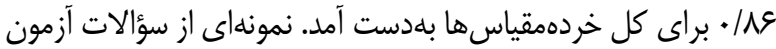

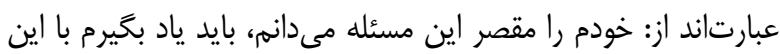
شرايط كنار بيايم، شرايط مىتوانست بهمراتب بدتر از اين باشد.

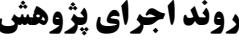

براى تقسيم دانشجويان به دو گروه با حساسيت بينفردى بالا و پايين، با توجه به اينكه يكى از روشهاى تعيين نقطهى برش برد

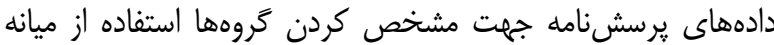

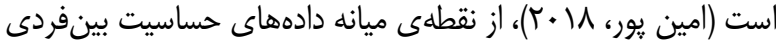

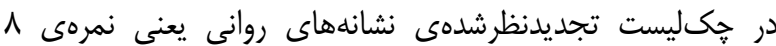

\begin{tabular}{|c|c|c|c|c|c|c|c|c|c|}
\hline \multirow{2}{*}{ معنادارى } & \multirow{2}{*}{$\begin{array}{l}\text { آزادى r } \\
\text { درجه }\end{array}$} & \multirow{2}{*}{ آزادى ا 1 آل } & \multirow{2}{*}{$\begin{array}{c}\text { آمارمى } \\
\text { F }\end{array}$} & \multirow{2}{*}{ لامبداى } & \multicolumn{2}{|c|}{ حساسيت بين فردى يايين } & \multicolumn{2}{|c|}{ حساسيت بينفردى بالا } & \multirow{2}{*}{ متغير } \\
\hline & & & & & انحراف معيار & ميانكين & انحراف معيار & ميانكين & \\
\hline $.1 .19^{m *}$ & r/9 & 1 & $\Delta / \Delta V$ &.$/ 948$ & $r / M$ & 1.199 & $4 / 19$ & $I V / \Delta V$ & سرزنش خود \\
\hline$\left.\cdot \cdot \cdot\right|^{*}$ & riq & 1 & $W / M P F$ &.$/ 9 \Delta \Delta$ & $r / \Lambda$ & $I T / T \Lambda$ & $.1 . .1$ & $11 / \cdot 0$ & يذيرش \\
\hline$\cdot \mid \cdot \cdot)^{*}$ & raq & 1 & TN/QAI &.$/ 918$ & T/Tr & $11 / 4$ & r/Q9 & או אוr/T & نشخوار فكرى \\
\hline$\cdot / \cdot \Delta^{* * *}$ & riq & 1 & $\left.\Gamma / \sim^{\prime} \omega\right)$ &.$/ 919$ & $r / q$ & $11 / 1$ & $r / \Delta$ & $11 / \cdot 1$ & تمر كز مجدد مثبت \\
\hline$\cdot / . . r^{* * *}$ & raq & 1 & Q/IrT &.$/ 990$ & $r / q r$ & (r/q & $r / v$ & $\mid r / q \pi$ & تمركز مجدد بر برنامهريزى \\
\hline.$/ .1 r^{* w}$ & riq & 1 & $s / r q u$ &.$/ 9 \vee \Delta$ & $r / \cdot V$ & $1 \% / 11$ & $4 / .1$ & $11 / 91$ & ارزيابى مجدد مثبت \\
\hline$\cdot / \wedge \Delta$ & riq & 1 & ( & $1 / \cdots$ & $r / \varphi q$ & $11 / \pi$ & $r / r \Delta$ & $11 / r$ & ديدكاهيرى \\
\hline$\cdot /\left.\cdot \cdot\right|^{*}$ & raq & 1 & $\Gamma \Delta / \wedge \Delta \Delta$ & $\cdot /$ AVA & $r / V T$ & $V / 99$ & $r / T$ & 1./ & فاجعلسازى \\
\hline.$/ . \cdot 1^{*}$ & raq & 1 & $r q / 4 \cdot q$ &.$/ 94 \mathrm{~V}$ & T/Ta & $V / A r$ & $r / r)$ & $9 / 91$ & سرزنش ديخران \\
\hline
\end{tabular}




\begin{tabular}{|c|c|c|c|c|c|c|c|}
\hline & & & كام تابع & ى از اصطلاحات & تشحيصى و بر & صdى تابع & جدول r \\
\hline معنادارى & درجهى آزادى & مجذور كاى & لامبداى ويلكز & همبستخى متعارف & درصد واريانس & مقدار ويزه & تعداد تابع \\
\hline $.1 . .1 *$ & 9 & $1) / \cdot \Delta$ & . IVAT & $\cdot / 0$ & $1 \ldots$ & . & 1 \\
\hline
\end{tabular}

بر اساس راهبردهاى تنظيم شناختى هيجان در كروه دانشجويان با

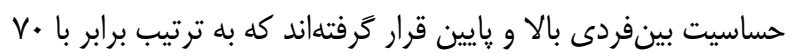

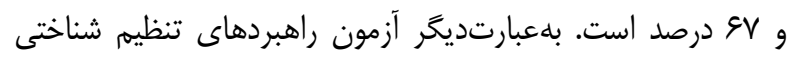

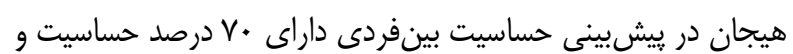

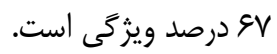
شكل ا جهت مقايسهى نيمرخ راهبردهاى تنظيم شناختى هيجان در

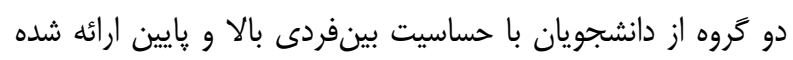

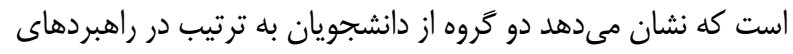

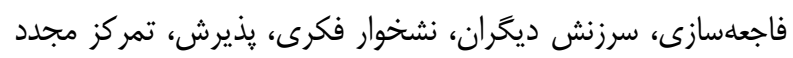

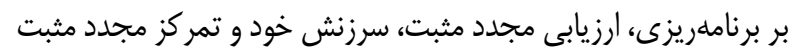

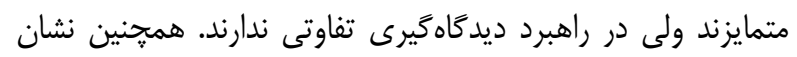

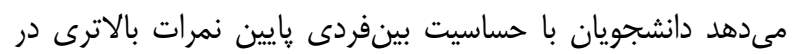

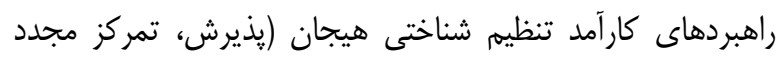

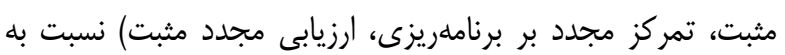

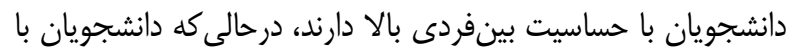

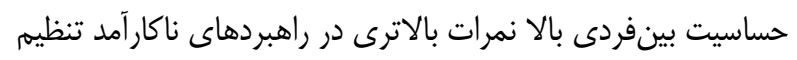

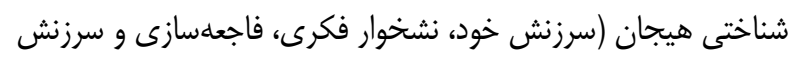

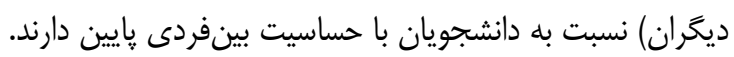

\section{بحث و نتيجه}

اين يزوهش با هدف تعيين نقش راهبردهاى تنظيم شناختى هيجان

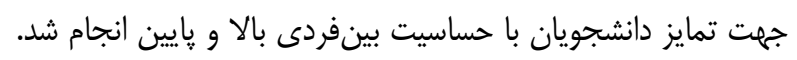

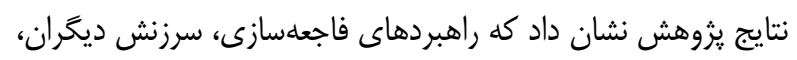

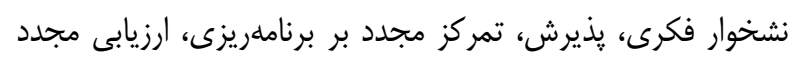

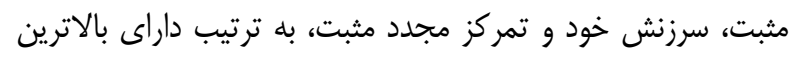

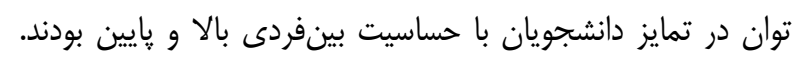

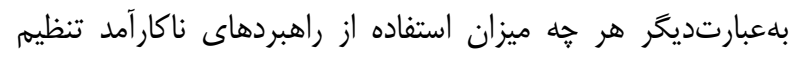

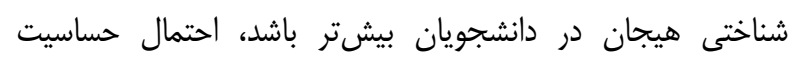

نتايج تحليل واريانس در جدول ا نشان مىدهد، دو گروهاز

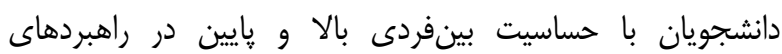

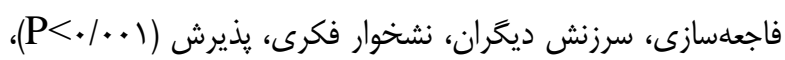

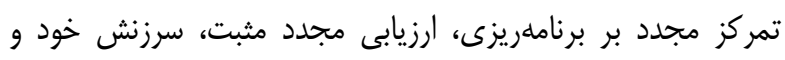

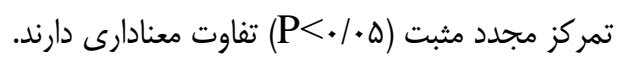

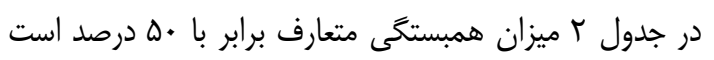

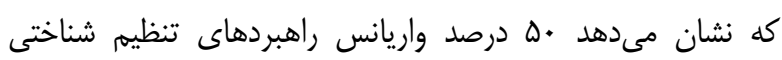

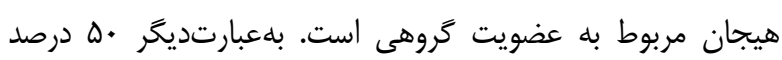

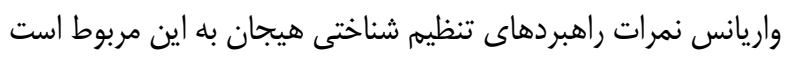

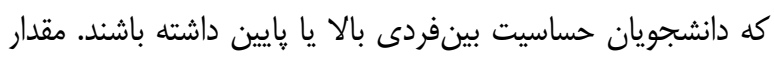

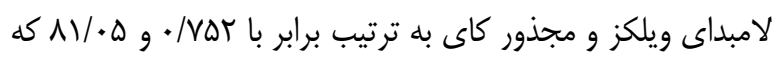

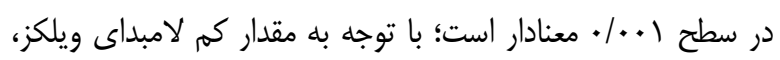

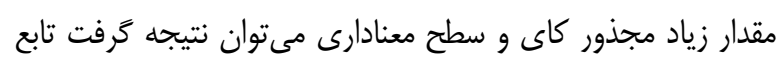

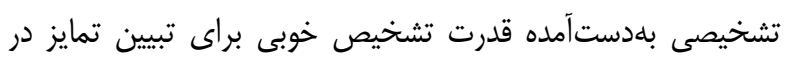

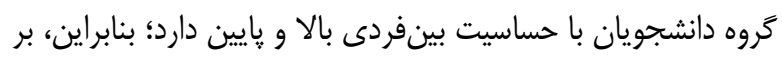

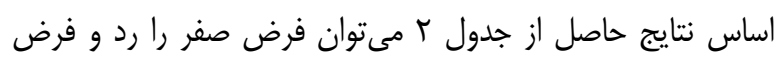

$$
\text { يثزوهش را تائيد كرد. }
$$

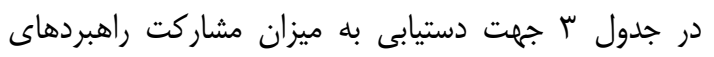

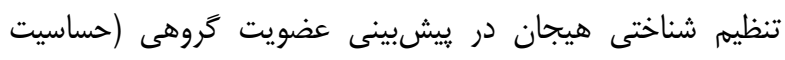

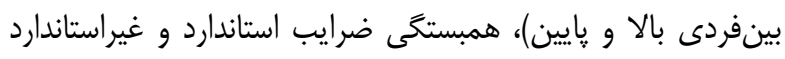

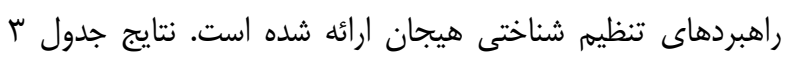

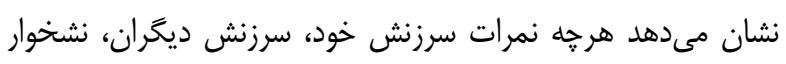

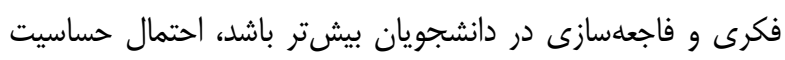

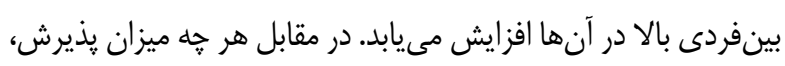

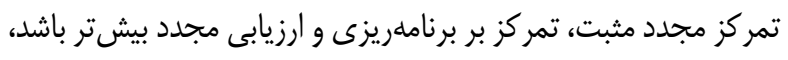

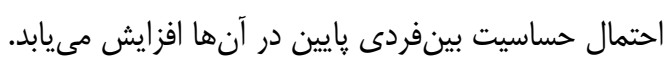

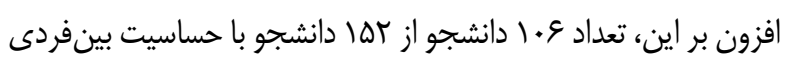
بالا و تعداد سو دانشجو از وسار دانشجو با حساسيت بينفردى بانيا بايين

جدول س. برآورد ضرايب استاندارد و غيراستاندارد در ييشيينى عضويت كَروهى

\begin{tabular}{|c|c|c|}
\hline ضرايب غيراستاندارد & ضرايب استاندارد & متغيرهاى ييشبين \\
\hline.$/ .14$ &.$/ .+\Delta$ & سرزنش خود \\
\hline$-. .1 . V r$ & שTr/.- & يذيرش \\
\hline.$/ 149$ & $\cdot \mid \times 11$ & نشخوار فكرى \\
\hline.$- / .+\wedge$ &.$- /$ MA & تمركز مجدد مثبت \\
\hline.$- / N F$ &.$- / \Delta T^{\prime} q$ & تمركز بر برنامهريزى \\
\hline.$- \cdot 1 \cdot 11$ & - & ارزيابى مجدد مثبت \\
\hline$-.1 .+q$ &.$- / 1 V$ & ديدكاه كيرى \\
\hline ./NT & TH Tא/. & فاجعه سازى \\
\hline .1 .91 & $\cdot / r$ & سرزنش ديگران \\
\hline
\end{tabular}




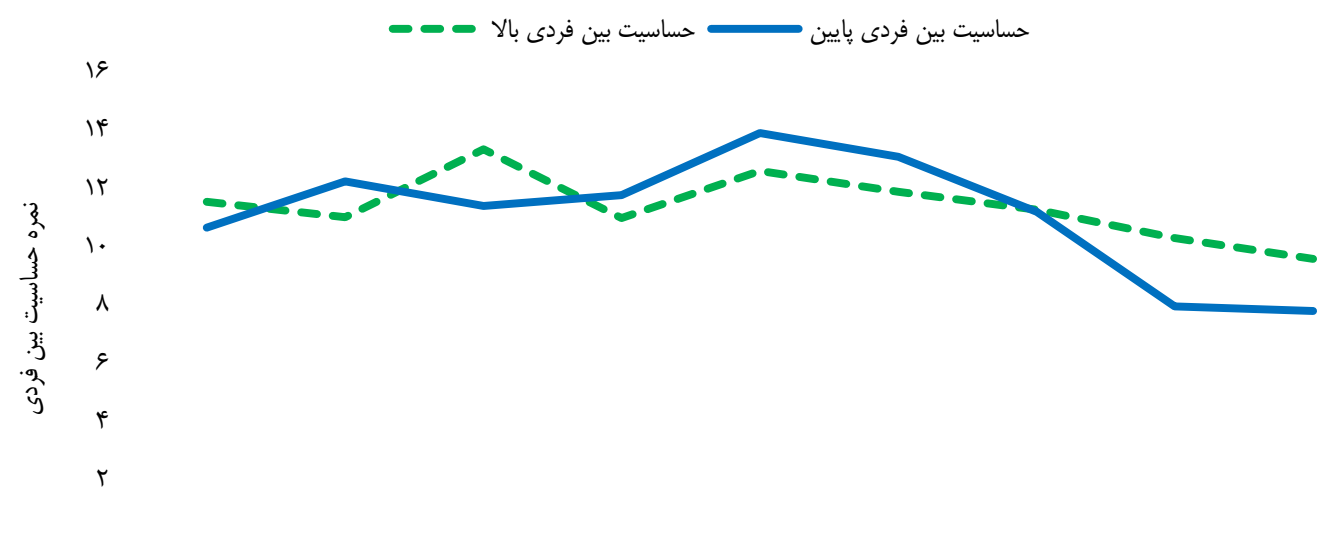

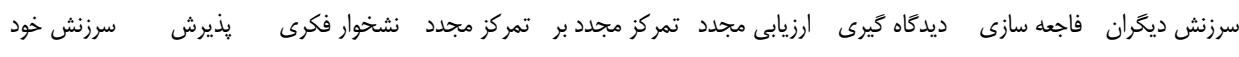

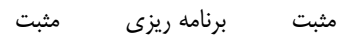

شكل ا. نيمرخ راهبردهاى تتظيم شناختى هيجان

و انتظارات بإينى از دريافت حمايت عاطفى از اطرافيان دارند و عموماً

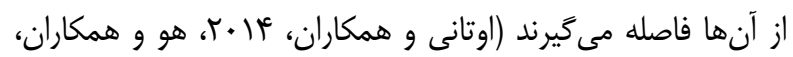

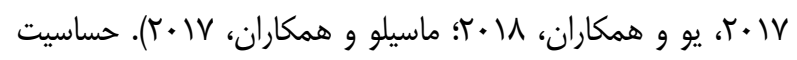

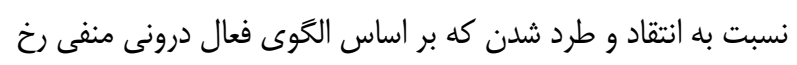

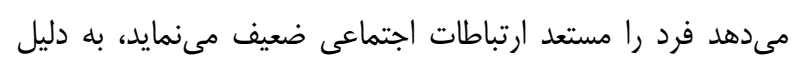

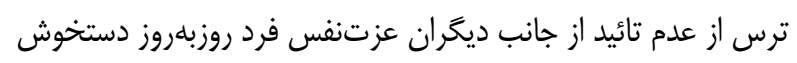

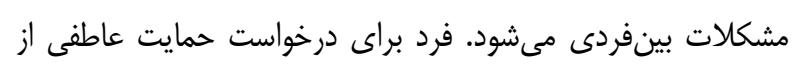

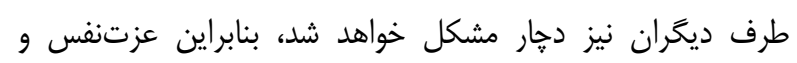

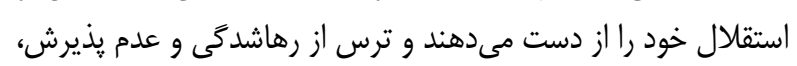

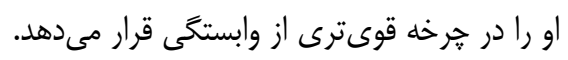

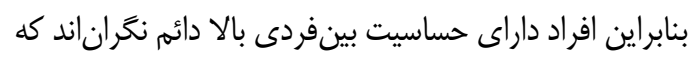

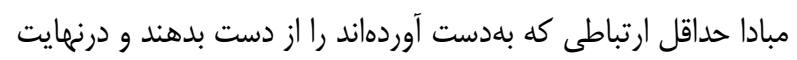

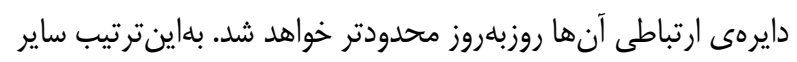

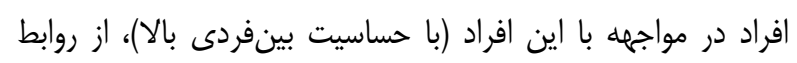

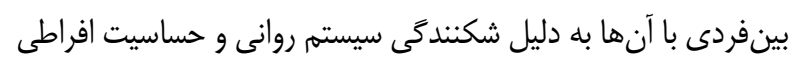

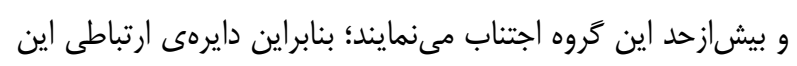

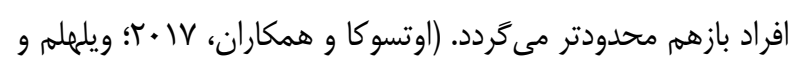

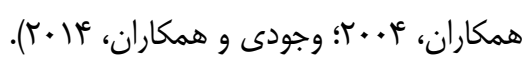
نكتهى قابل بحث ديكر هيجانات و خُلق منفى افراد دار داراى

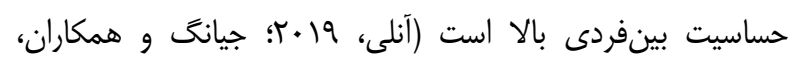

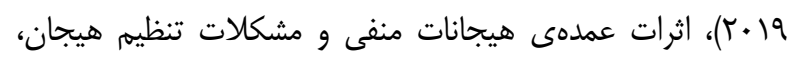

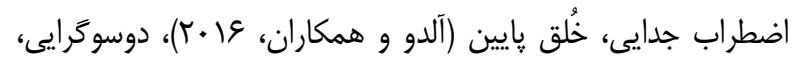

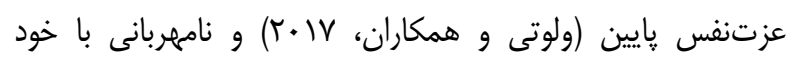

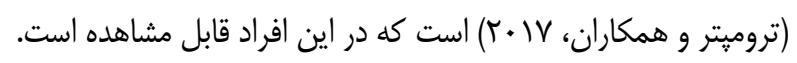

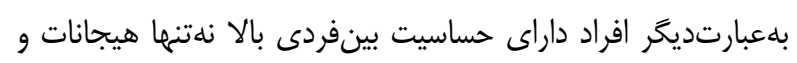

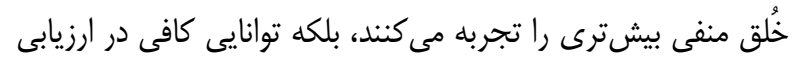

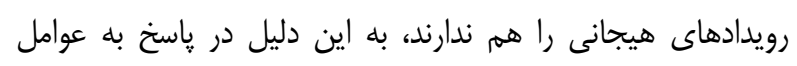

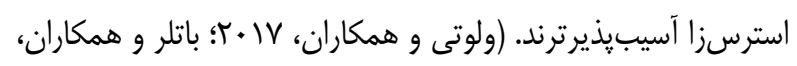

. (T+V

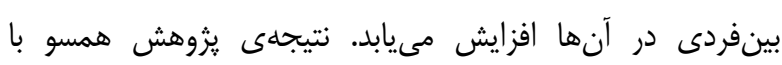

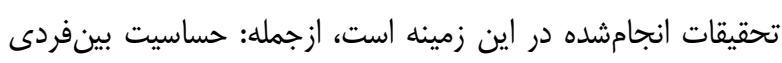

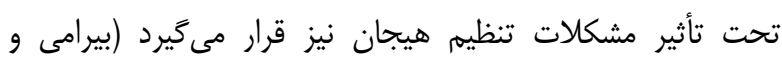

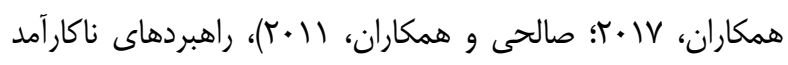

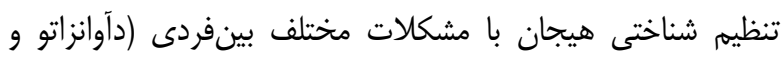

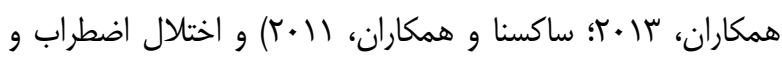

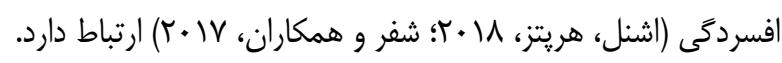

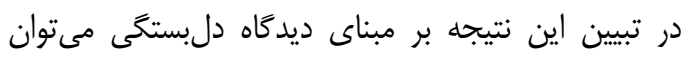

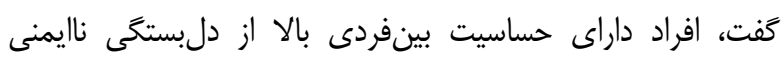

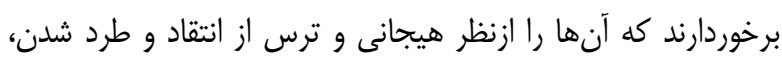

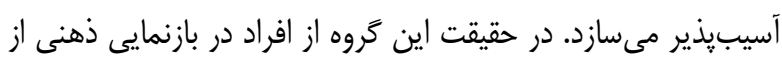

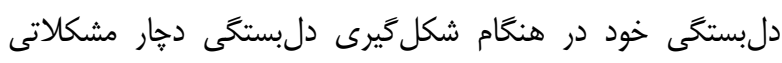

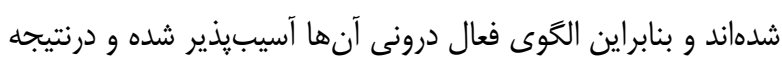

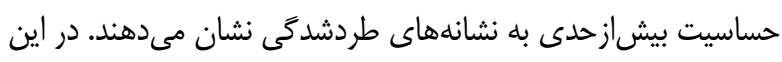

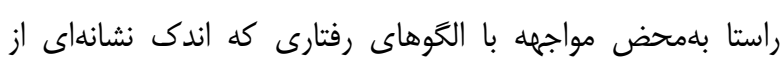

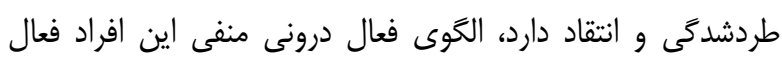

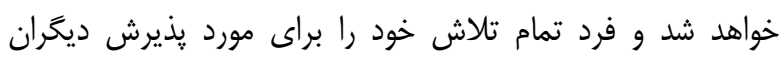

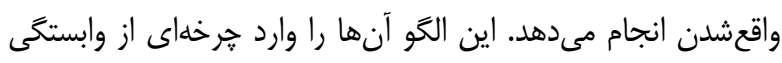

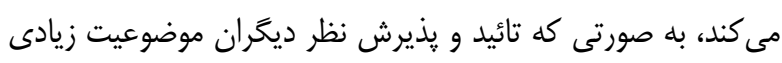

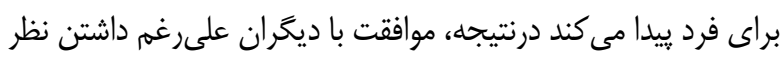

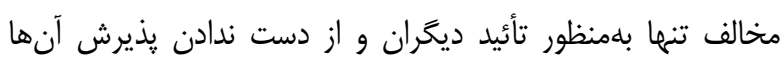

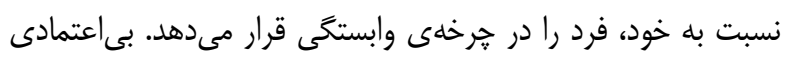

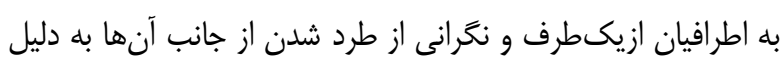

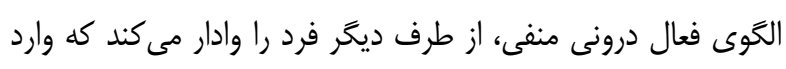

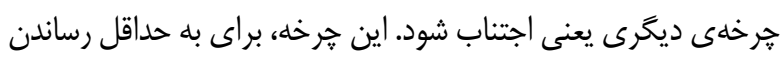
ناكامى شكل مى خيرد.

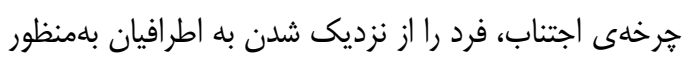

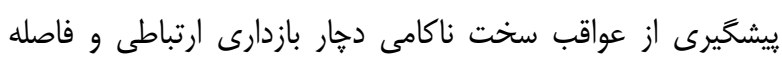

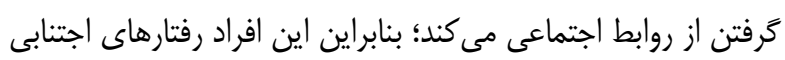


راهبردهاى كارآمد تنظيم شناختى استفاده مى كنند، كاهش مى يابد.

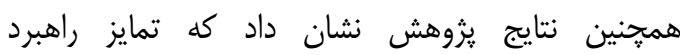
ديدكاهگيرى در دو كروه از دانشجويان با حساسيت بينفردى بنان بالا و

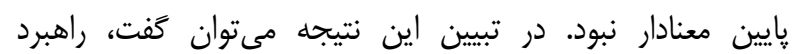

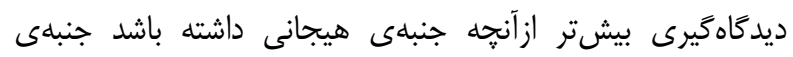

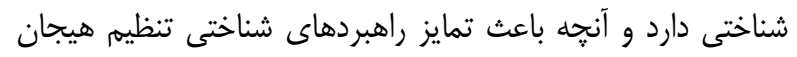

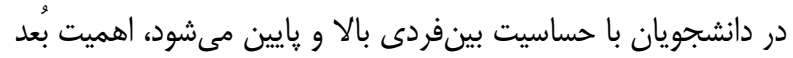

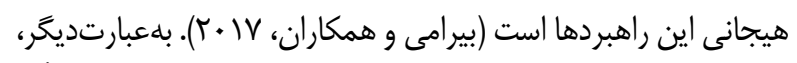

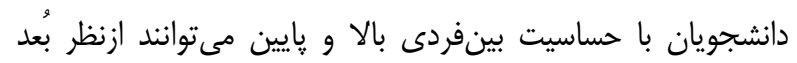

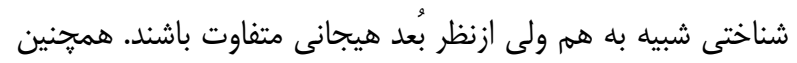

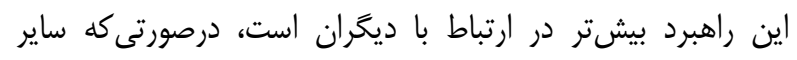

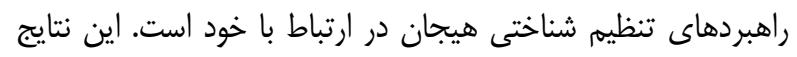

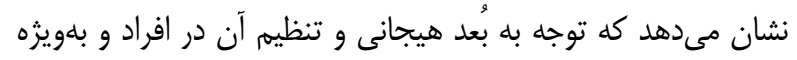

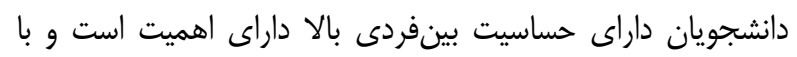

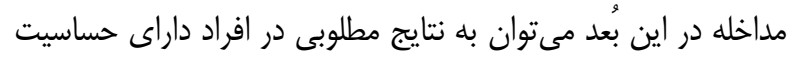
بينفردى بالادست يافت.

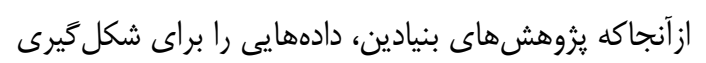

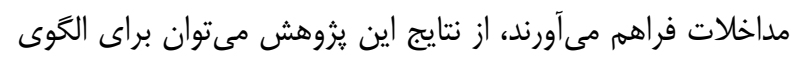

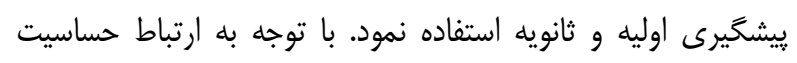
بينفردى و راهبردهاى تنظيم شناختى هيجان، در سطح بيشخيرى

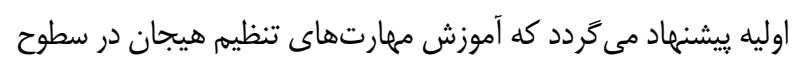

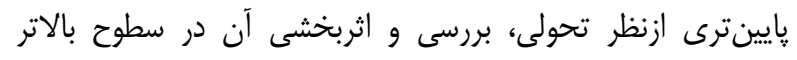

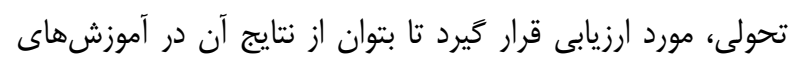

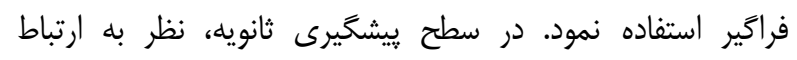

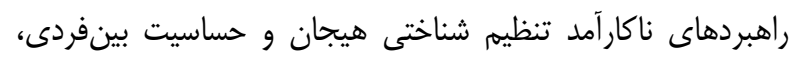

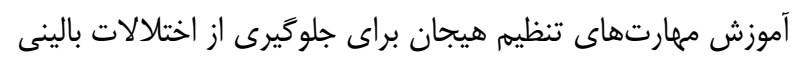

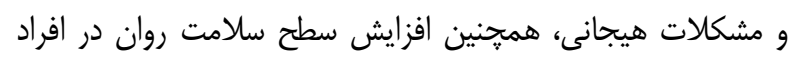

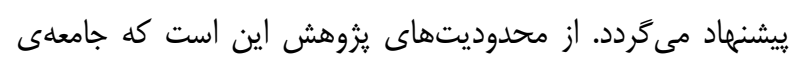

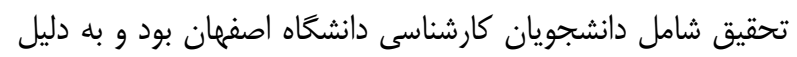

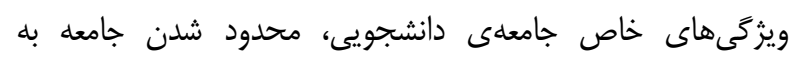
دانشجويان ممكن است تعميميذيرى نتايج را تحت تأثير قرار دهدئ
بنابراين دانشجويان با حساسيت بين فردى بالا در مقايسه با

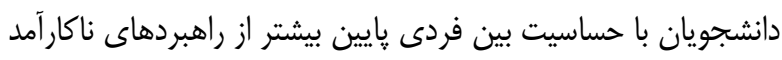

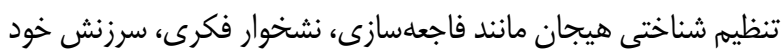

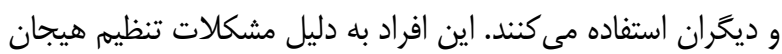

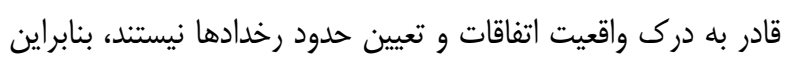

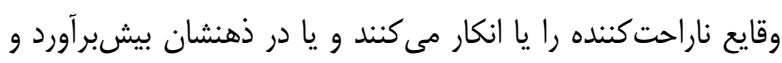

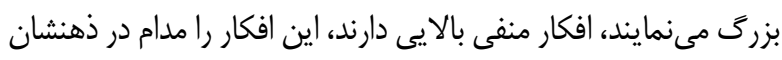

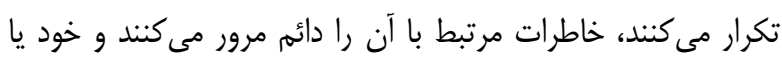

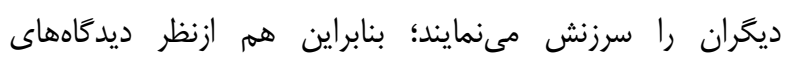

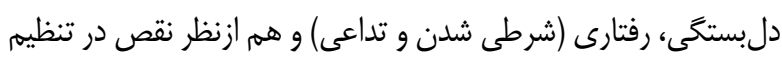

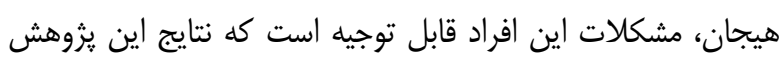
نيز توياى آن است.

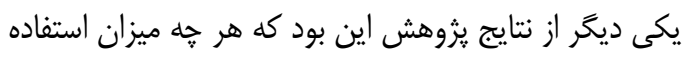

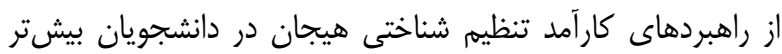

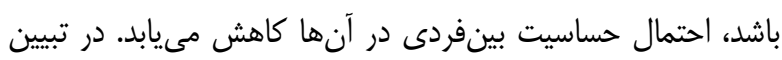

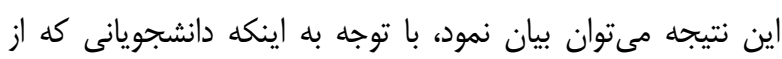

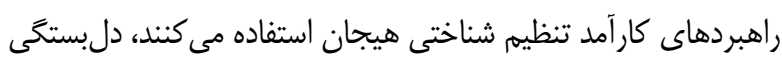

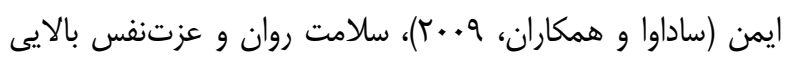

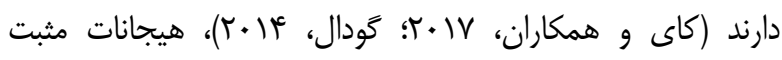

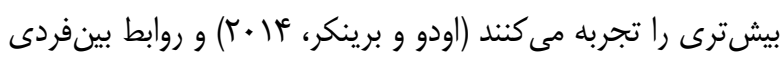

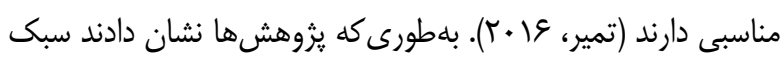

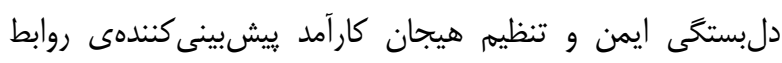

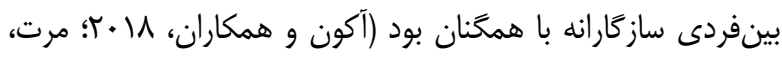

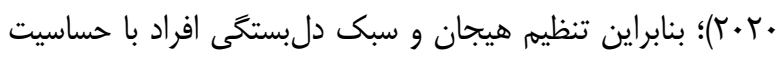

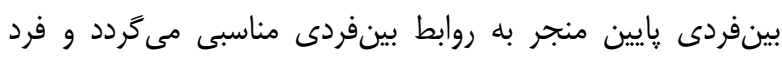
توانايى مقبوليت اجتماعى به دست مى بـآورد.

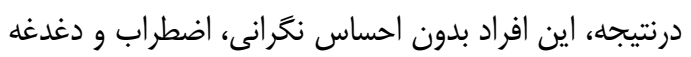

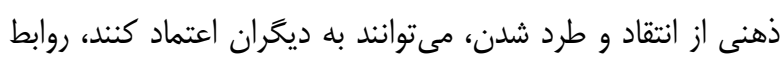

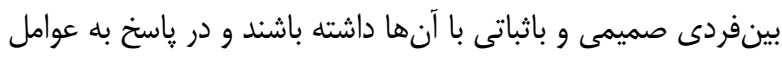

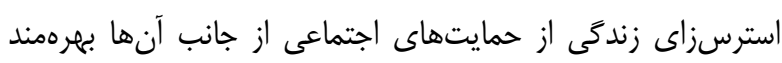

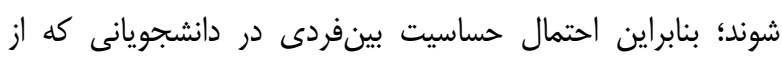

\section{Reference}

Akün, E., Durak-Batıgün, A., Devrimci-Özgüven, H. \& Baskak, B. (2018). Positive symptoms and perceived parental acceptance-rejection in childhood: socioeconomic level and regulatory role of gender. Turkish Psychiatry Journal, 29(2), 109-115. [DOI:10.5080/u22681] [PMid:302158 39]
Aldao, A., Gee, D. G., De Los Reyes, A., \& Seager, I. (2016). Emotion regulation as a transdiagnostic factor in the development of internalizing and externalizing psychopathology: Current and Future Directions. Development and Psychopathology, 28(4pt1), 927-46. [DOI:10. 1017/S0954579416000638] [PMid:27739387] 
Aminpour, H. (2018). Descriptive Statistics in Psychology and Educational Sciences (Psychology and Educational Sciences).12th ed. Tehran: Payame Noor University. [In Persian, 1397]

Anli, G. (2019). Investigating the relationship between sense of classroom community and interpersonal sensitivity. International Journal of Progressive Education, 15(5), 371-9. [DOI:10.29329/ijpe. 2019.212.24]

Anisi, j., Eskandari, M., Bahmanabadi, S., Noohi, S., \& Tavalayi, A. (2014/1393). Standardization of Symptom Checklist 90 Revised (SCL-90 -R) of a military Unit. Journal of Military Psychology, 5(17), 57-67. [In Persian]

Barlett, J. E., Kotrlik, J. W., \& Higgins, C. C. (2001). Organizational research: Determining appropriate sample size in survey research. Information Technology, Learning, and Performance Journal, 19(1), 43-50.

Bayrami, M., Hashemi, T., \& Ashouri, M. (2017/1396). Effectiveness of Emotional Regulation Training on Students Social Adaptation and Interpersonal Sensitivity. Quarterly social psychology research, 27(7), 1-14. [In Persian]

Blalock, D., Kashdan, T., \& Farmer, A. (2016). Trait and Daily Emotion Regulation in Social Anxiety Disorder. Cognitive Therapy and Research, 40(3), 416-425. [DOI:10.1007/s10608-015-9739-8]

Butler, J. C., Doherty, M. S., Potter, R. M. (2007). Social antecedents and consequences of interpersonal rejection sensitivity. Personality and Individual Differences, 43(6), 1376-85. [DOI:10. 1016/j.paid.2007.04.006]

CAI, R. Y., Richdale, A. L., Dissanayake, C., \& Uljarevic, M. (2017). Brief report: interrelationship between emotion regulation, intolerance of uncertainty, anxiety, and depression in youth with autism spectrum disorder. Journal of Autism and Developmental Disorders, 48(1), 316325. [DOI:10.1007/s10803-017-3318-7] [PMid: 28980172]

D’Avanzato, C., Joormann, J., Siemer, M., \& Gotlib, I. H. (2013) Emotion regulation in depression and anxiety: examining diagnostic specificity and stability of strategy use. Cognitive Therapy and Research, 37(5), 968-80. [DOI:10.1007/s10608013-9537-0]

Dryman, M. T., \& Heimberg, R. G. (2018). Emotion regulation in social anxiety and depression: a systematic review of expressive suppression and cognitive reappraisal. Clinical Psychology Review, 65, 17-42. [DOI:10.1016/j.cpr.2018.07. 004] [PMid:30064053]

Extremera, N., Sánchez-Álvarez, N., \& Rey, L. (2020). Pathways between ability emotional intelligence and subjective well-being: bridging links through cognitive emotion regulation trategies. Sustainability, 12, 2-11. [DOI:10.3390/su12052 111]

Farahani, S., Rafiepoor, A., \& Jafari, T. (2019). The Role of Mediator of Cognitive Emotion Regulation Strategies in the Relationship between Mindfulness and Perceived Stress in People with
High Blood Pressure. International journal of applied behavioral sciences, 6(2), 52-9.

Gall, M., Borg, W., \& Gall, J. (2015/1394). Quantitative and Qualitative Research Methods in Educational Sciences and Psychology Collective. Trans. Nasr AR. 9th ed. Tehran: Shahid Beheshti University. [In Persian]

Garnefski, N., Kraaij, V. (2014). Bully victimization and emotional problems in adolescents: moderation by specific cognitive coping strategies? Journal of Adolescence, 37(7), 115360.

Gilbert, K., Mineka, S., Zinbarg, R. E., Craske, M. G., \& Adam, E. K. (2016). Emotion regulation regulates more than emotion: associations of momentary emotion regulation with diurnal cortisol in current and past depression and anxiety. Clinical Psychological Science, 5(1), 37-51. [DOI: 10.1177/2167702616654437] [PMid: 28944106 PMCid: PMC5606207]

Golmahammadi, K., Sadeghi, H., Shamsoldini, H, Zohrabi, S., \& Hashemi, F. (2016/1395). The simultaneity investigation of role of anxiety sensitivity and emotional regulation in prediction of social anxiety disorder. Journal of health Breeze Family Health Summer, 5(1), 40-6. [In Persian]

Goodall, K. (2014). Individual differences in the regulation of positive emotion: The role of attachment and self esteem. Personality and Individual Differences, 74(2015), 208-13. [DOI: 10.1016/j.paid.2014.10.033]

Gross, J. J. (2015). Emotion regulation: Current status and future prospects. Psychological Inquiry, 26(1), 1-26. [DOI:10.1080/1047840X.2014.940781]

Hassani, J., \& Ariana Kiaa, E. (2016). Cognitive emotion regulation strategies, anxiety and impulsivity in bipolar disorder with and without comorbid obsessive-compulsive disorder. Iranian Journal of Psychiatry and Clinical Psychology, 22(1), 39-49. [In Persian]

Harb, G.C., Heimberg, R.G., Fresco, D.M., Schnrier, F.R. \& Liebowitz, M.R. (2002). The psychometric properties of the interpersonal sensitivity measure in social anxiety disorder. Behaviour Research and Therapy, 40(8), 961-79. [DOI:10.1016/ S00057967(01)00125-5]

Hopwood, C. J., \& Good, E. W. (2018). Structure and correlates of interpersonal problems and sensitivities. Journal of Personality, 87(4),1-13, 843-855. [DOI:10.1111/jopy.12437] [PMid:303 20881]

Huh, H. J., Kim, K. H., Lee, H. K., \& Chae, J. H. (2017). The relationship between childhood trauma and the severity of adulthood depression and anxiety symptoms in a clinical sample: The mediating role of cognitive emotion regulation strategies. Journal of Affective Disorders, 213, 44-50. [DOI:10.1016/ j.jad.2017.02.009] [PMid:28189964]

Jiang, D., Hou, Y., Chen, X., Wang, R., Fu, C., Li, B., \& Liu, X. (2019). Interpersonal sensitivity and loneliness among Chinese Gay Men: A Crosssectional survey. International Journal of Environmental Research and Public Health, 16(11), 1-14. [DOI:10.3390/ijerph16112039] 
[PMid:31181720 PMCid:PMC6603862]

Kerlinger, F.N., \& Pedhazur. E. (2018). Multiple regression in behavioral research $\mathrm{H}$, Trans. Saraai H. 9th Ed, Tehran: Samt. [In Persian, 1397]

Kever, A., Pollatos, O., Vermeulen, N., \& Grynberg, D. (2015). Interoceptive sensitivity facilitates both antecedent- and response-focused emotion regulation strategies. Personality and Individual Differences, 87, 20-23. [DOI:10.1016/j.paid.2015. 07.014]

Lei, H., Zhang, X., Cai, L., Wang, Y., Bai, M., \& Zhu, $X$. (2014). Cognitive emotion regulation strategies in outpatients with major depressive disorder. Psychiatry Research, 218(1), 87-92. [DOI:10. 1016/j.psychres.2014.04.025] [PMid:24774074]

Masillo, A., Valmaggia, L., Saba, R., Brandizzi, M., Lo Cascio, N., Telesforo, L., Fiori Nastro, P. (2017). Interpersonal sensitivity, bullying victimization and paranoid ideation among help-seeking adolescents and young adults. Early Intervention in Psychiatry, 13, 57-63. [DOI:10.1111/eip. 12447] [PMid:28560857]

Mert, A. (2020). Attachment Styles and Emotion Regulation as Predictions of Peer Relations in Secondary School Students. Education, 140(4), 194-206.

Modabernia, M.J., Shojaie Tehranie, H., Falahi, M., \& Faghirpour, M. (2010). Normalizing SCL-90-R Inventory in Gilan High School Students. Journal of Guilan University of Medical Sciences, 19(75), 58-65. [In Persian, 1389]

Najjarpoor, E., Ghanbarih, B., \& mazaheri, M. (2017). The effectiveness of short-term subject-based psychotherapy on reducing interpersonal sensitivity, interpersonal ringing, and the need for confirmation in people with avoidant personality trait: A single-subject study. Quarterly of Counseling Culture and Psychotherapy, 8 (31), 79104. [In Persian, 1396]

Nyström, M., Kjellberg, E., Heimdahl, U., \& Jonsson, B. (2018). Shame and interpersonal sensitivity: Gender differences and the association between internalized shame coping strategies and interpersonal sensitivity. Bulletin of the Menninger Clinic, 82(2), 137-55. [DOI:10.1521/ bumc.2018.82.2.137] [PMid:29791193]

Odou, N., \& Brinker, J. (2014). Exploring the relationship between rumination, self-compassion, and mood. Self and Identity, 13(4), 449-59. [DOI:10.1080/15298868.2013.840332]

Otani, K., Suzuki, A., Matsumoto, Y., Shibuya, N., Sadahiro, R., \& Enokido, M. (2014). Correlations of interpersonal sensitivity with negative working models of the self and ther: evidence for link with attachment insecurity. Annals of General Psychiatry, 13(1), 5-13. [DOI:10.1186/1744-859X -13-5] [PMid:24529211 PMCid:PMC3925358]

Otsuka, A., Takaesu, Y., Sato, M., Masuya, J., Ichiki, M., Kusumi, I., \& Inoue, T. (2017). Interpersonal sensitivity mediates the effects of child abuse and affective temperaments on depressive symptoms in the general adult population. Neuropsychiatric Disease and Treatment, 13, 2559-68.
[DOI:10.2147/NDT.S144788] [PMid: 29042786 PMCid: PMC5634385]

Sadava, S. W., Busseri, M. A., Molnar, D. S., Perrier, C. P. K., \& Decourville, N. (2009). Investigation of a four pathway model of adult attachment orientation and health. Journal of Social and Personal Relationships, 26(5), 604-33. [DOI:10.1177/0265407509354402]

Sadri Damirchi, E., Ghazivaloyi, F., Shishegaran, S., \& Mohammadi, N. (2018). Comparison of the Cognitive Emotion Regulation, Locus of Control and Meaning in life in Native and Non-Native Students. Journal of Research on Religion \& Health, 4(5), 5- 16. [In Persian, 1397]

Salehi A, Baghban I, Bahrami F, Ahmadi A. (2011). The effect of emotion regulation training based on dialectical behavior therapy and gross process model on symptoms of emotional problems. Zahedan Journal of Resesearch in Medical Sciences, 14(2), 49-55. [In Persian, 1390]

Salehi, A., Mazaheri, Z., Aghajani, Z., \& Jahanbazi, B. (2015). The Role of Cognitive Emotion Regulation Strategies in the Prediction of Depression. Knowledge \& Research in Applied Psychology, 1(16), 108-17. [In Persian, 1394]

Saxena, P., Dubey, A., \& Pandey, R. (2011) .Role of emotion regulation difficulties in predicting mental health and well-being. Journal of Projective Psychology and Mental Health, 8(1), 147-55.

Schäfer, J. Ö., Naumann, E., Holmes, E. A., TuschenCaffier, B., \& Samson, A. C. (2017). Emotion regulation strategies in depressive and anxiety symptoms in youth: A meta-analytic review. Journal of Youth and Adolescence, 46(2), 261-76. [DOI:10.1007/s10964-016-0585-0] [PMid:27734198]

Schnell, K., \& Herpertz, S. (2018). Emotion Regulation and Social Cognition as functional targets of mechanism-based psychotherapy in major depression with comorbid personality pathology. Journal of Personality Disorders, 32, 12-35. [DOI:10.1521/pedi.2018.32.supp.12] [PMid:29388896]

Slanbekova, G. K., Chung, M. C., Karipbaev, B. I., Sabirova, R. S., \& Alimbayeva, R. T. (2019). Posttraumatic Stress and Interpersonal Sensitivity: Alexithymia as Mediator and Emotional Expressivity as Moderator. Psychiatric quarterly, 90 (1), 249-261. [DOI:10.1007/s11126-018-9612-5] [PMid:30515699]

Southward, M., Altenburger, E., Moss, S., Cregg, D., \& Cheavens, J. (2018). Flexible, Yet firm: A model of healthy emotion regulation. Journal of Social and Clinical Psychology, 37(4), 231-51. [DOI:10.1521/jscp.2018.37.4.231] [PMid:30337772 PMCid:PMC6191057]

Stikkelbroek, Y., Bodden, D. H., Kleinjan, M., Reijnders, M., \& van Baar, A. L. (2016). Adolescent depression and negative life events, the mediating role of cognitive emotion regulation. PLoS One, 11(8), 1-16. [DOI:10.1371/journal. pone.0161062] [PMid:27571274 PMCid:PMC50 03336] 
Suveg, C., Jacob, M., \& Payne, M. (2010). Parental interpersonal sensitivity and youth social problems: A mediational role for child emotion dysregulation. Journal of Child and Family Studies, 19(6), 677-86. [DOI:10.1007/s10826-010 $-9354-y]$

Tamir, M. (2016). Why do people regulate their emotions? A taxonomy of motives in emotion regulation. Personality and Social Psychology Review, 20(3), 199-222. [DOI:10.1177/10888683 15586325] [PMid:26015392]

Tully, E. C., Ames, A. M., Garcia, S. E., \& Donohue, M. R. (2015). Quadratic associations between empathy and depression as moderated by emotion dysregulation. The Journal of Psychology, 00(0), 1-25. [DOI:10.1080/00223980.2014.992382] [PMid:25565484]

Turliuc, M.N., \& Bujor, L. (2013). Emotional regulation, expansion of the concept and its explanatory models. Models. Applied social sciences psychology. Physical Education and Social Medicine, 1, 99-106.

Trompetter, H.R., de Kleine, E., \& Bohlmeijer, E.T. (2017). Why does positive mental health buffer against psychopathology? An exploratory study on self-compassion as a resilience mechanism and adaptive emotion regulation strategy. Cognitive Therapy and Research, 41(3), 459-68. [DOI: 10.1007 / s10608-016-9774-0] [PMid: 28515539
PMCid: PMC5410199]

Velotti, P., Garofalo, C., Bottazzi, F., \& Caretti, V. (2017). Faces of shame: implications for selfesteem, emotion regulation, aggression, and wellbeing. The Journal of Psychology, 151(2), 171-84. [DOI:10.1080/00223980.2016.1248809] [PMid:27858531]

Vidyanidhi, K., Sudhir, P. M. (2009). Interpersonal sensitivity and dysfunctional cognitions in social anxiety and depression. Asian Journal of Psychiatry, 2 (1), 25-8. [DOI:10.1016/j.ajp.2008. 12.001] [PMid:23051014]

Vojudi, B., Otared, N., \& Poursharifi, H. (2014). on the comparsion of Interpersonal sensitivity and assertiveness between drug-dependent persion and ordinary people. Quarterly Journal of Research on addiction, 8(31), 109-18. [In Persian, 1393]

Wilhelm, K., Boyce, P., Brownhill, S. (2004). The relationship between interpersonal sensitivity, anxiety disorders and major depression. Journal of Affect disorder, 79(1-3), 33-41. [DOI:10.1016/ S0165-0327(02)00069-1]

You, Z., Zhang, Y., Zhang, L., Xu, Y., \& Chen, X. (2018). How does self-esteem affect mobile phone addiction? The mediating role of social anxiety and interpersonal sensitivity. Psychiatry Research, 271, 526-31. [DOI:10.1016/j.psychres.2018.12. 040] [PMid:30553099] 